\title{
Organerhaltende Therapie des Harnblasenkarzinoms
}

\author{
Jürgen Dunst ${ }^{1}$ \\ Online publiziert: 27. April 2020 \\ c C Der/die Autor(en) 2020
}

Hintergrund Seit Jahrzehnten besteht die Möglichkeit einer organerhaltenden Behandlung des muskelinvasiven Harnblasenkarzinoms durch transurethrale Tumorresektion (TUR) und Radiochemotherapie (RCT). In den Patienteninformationen der Deutschen Krebshilfe (den „Blauen Ratgebern") wird diese Therapie als äquieffektive Alternative zur Zystektomie dargestellt [4]. Dennoch machen die Urologen von dieser Option wenig Gebrauch. Dass das an den Patienten liegt, die eine Zystektomie als primäre Therapie bevorzugen, ist kaum vorstellbar. Vermutlich besteht einfach ein Informationsdefizit der behandelnden Ärzte. Es ist daher wichtig, immer wieder auf dieses Therapiekonzept, das 1982 von den Erlangern Alfred Sigel (Urologie) und Rolf Sauer (Radioonkologie) eingeführt wurde, hinzuweisen und aktuelle Daten zu publizieren, zumal der zusätzliche Einsatz der regionalen Tiefenhyperthermie zur RCT einen Prognosesprung verspricht. Die Kollegen des Universitätsklinikums Erlangen machen das und haben kürzlich überzeugende Langzeitdaten vorgelegt [7].

Patienten und Methode Zwischen 1982 und 2016 wurden an der Strahlenklinik der Universität Erlangen-Nürnberg 664 Patienten mit einem Blasenkarzinom lediglich noch transurethral reseziert, also primär nicht mehr zystektomiert, sondern mit dem Ziel des Organerhalts anschließend nur noch bestrahlt, später radiochemotherapiert und in den letzten Jahren noch zusätzlich hyperthermiert. Für die hier kommentierte Analyse wurden die Therapieergebnisse bei 369 Patienten mit oberflächlichen High-risk-Tumoren (pTa, pTis, pT1, jeweils mit Indikation zur Zystektomie) und T2-Tumoren untersucht, fortgeschrittene Tumoren (cT3-4) \footnotetext{
cancer (Ta, Tis, T1, T2). The Oncologist 24:1-10

Prof. Dr. med. Jürgen Dunst

Juergen.Dunst@uksh.de

1 Klinik für Strahlentherapie, Campus Kiel, Universitätsklinikum Schleswig-Holstein, Feldstr. 21, 24105 Kiel, Deutschland
}

Originalpublikation Merten R, Ott O, Haderlein M et al (2019)

Long-term experience of chemoradiotherapy combined with deep regional hyperthermia for organ preservation in high-risk bladder aber ausgeschlossen. Ziel war eine organ- und funktionserhaltende Therapie bei Patienten mit der herkömmlichen Indikation zur Zystektomie.

Das Therapiekonzept bestand aus einer transurethralen Resektion (TUR mit dem Ziel einer R0-TUR) und einer Radiotherapie sowie anschließenden Salvage-Zystektomie bei Rest- oder Rezidivtumor. Die Radiotherapie war von 1982 bis 1985 eine alleinige Strahlentherapie (RT), ab 1985 eine simultane Radiochemotherapie (RCT) mit Cisplatin, später zusätzlich 5-FU, also eine nur strahlensensibilisierende Chemotherapie während der Radiotherapie (keine neoadjuvante oder adjuvante Chemotherapie). Die Strahlendosis betrug 50,4Gy im Bereich der Blase und der regionalen Lymphknoten in Einzeldosen von 5-mal wöchentlich 1,80 Gy, gefolgt von einem Boost auf die Harnblase bis 55,8 Gy nach R0-TUR bzw. bis zu einer Gesamtdosis von 59,4Gy bei Residualtumor (R1-2 nach TUR). Ab 2005 wurde während der Strahlentherapie zusätzlich 1-mal wöchentlich eine regionale Tiefenhyperthermie mit einem BSD-2000-3D/PC-Hyperthermie-System (RCT + RHT) durchgeführt. Das mediane Follow-up betrug 71 Monate.

Ergebnisse 290 von 369 Patienten (83\%) erlebten eine komplette Tumorremission bei der Kontroll-TUR 6 Wochen nach Radiotherapie. Die CR-Rate betrug 68\% nach alleiniger Radiotherapie, 86\% nach Radiochemotherapie und $87 \%$ nach Radiochemotherapie plus Hyperthermie ( $p=0,037)$. Das 5-Jahres-Überleben betrug $45 \%$ nach RT, $64 \%$ nach RCT und $87 \%$ nach RCT + RHT. 55 Patienten wurden im Verlauf v.a. wegen Rezidiv oder Zweittumor zystektomiert. Nach 5 Jahren wurde ein Blasenerhalt bei $82 \%$ der Patienten nach Radiochemotherapie und bei $96 \%$ nach Radiochemotherapie plus Hyperthermie erreicht; nach 10 Jahren betrugen diese Werte $78 \%$ bzw. 96\%. Die meisten Patienten vertrugen die Therapie (RT oder RCT) gut. Eine Akuttoxizität Grad 3 und 4 betraf fast nur hämatologische Nebenwirkungen (Leukopenie Grad 3: 25\%, Thrombopenie: 6\%, Grad 4: Leukopenie $4 \%$ ). Die Grad-3-Nebenwirkungen der Strahlentherapie als 
radiogene Zystitis betrafen $6 \%$ der Patienten. Die Spättoxizität war gering: $61 \%$ Grad 0 bei RCT, $32 \%$ Grad 0 bei RCT + RHT. Relevante Spätfolgen betrafen Zystektomien wegen Blasenschrumpfung $(2 \%, n=6)$ und eine reduzierte Blasenkapazität <200 ml (10\%), 5 Patienten entwickelten operationspflichtige Darmstenosen.

Schlussfolgerungen der Autoren Die multimodale organund funktionserhaltende Therapie, bestehend aus TUR und Radiochemotherapie, ist bei Harnblasenkarzinomen sehr erfolgreich. Die erreichten Überlebensraten sind zumindest so gut wie nach Zystektomie. Dabei können 4 von 5 Patienten eine funktionsfähige Blase behalten. Die Hinzunahme der regionalen Tiefenhyperthermie verbessert die Erfolgsaussicht auf einen Blasenerhalt zusätzlich.

\section{Kommentar}

„Radiotherapy and organ preservation in bladder cancer: are we ignoring the evidence?" [3]. Dieser immer noch lesenswerte Kommentar von Mary Gospodarowicz erschien im Jahr 2002 im Journal of Clinical Oncology anlässlich einer Publikation der Erlanger Daten über die organerhaltende Behandlung des Harnblasenkarzinoms [8]. Bereits damals stand fest, dass die organerhaltende Therapie bei muskelinvasiven Blasenkarzinomen als Alternative zur Zystektomie angesehen werden muss ohne Einbußen im ,overall survival". Es gab damals (wie heute) keine direkten randomisierten Vergleiche zwischen Zystektomie und dem organerhaltenden Konzept. Aber die beste verfügbare Evidenz zeigte und zeigt die Gleichwertigkeit beider Ansätze bezüglich des Überlebens [1, 2, 9]. In den blauen Ratgeberheften der Deutschen Krebshilfe wird die Möglichkeit der Organerhaltung dargestellt [4]. Dennoch wird diese Option von den Urologen kaum genutzt. Es ist erstaunlich, wie wenig sich daran in fast 20 Jahren geändert hat!

Zurück zum Anfang: Das Urothelkarzinom der Harnblase (nur darum geht es; die anderen seltenen Entitäten sind gesondert zu betrachten) ist ein strahlenempfindlicher Tumor. In den 1970er- und 1980er-Jahren wurden an einigen Orten (v.a. in Großbritannien und Skandinavien) hoch dosierte Bestrahlungen als Alternative zur Zystektomie eingesetzt. Die Ergebnisse dieser ,radikalen Radiotherapie“ hinsichtlich Tumorkontrolle und Überleben waren nicht schlecht, aber es gab eine relevante Rate an Spätfolgen mit schlechtem funktionellem Ergebnis. Vor allem eine Blasenschrumpfung mit stark verkürztem Miktionsintervall, die gelegentlich eine sekundäre Zystektomie erforderte. Eine wesentliche Verbesserung (aus meiner Sicht der entscheidende Durchbruch) waren die ab Ende der 1980er-Jahre in Boston und Erlangen entwickelten Konzepte, die die Grundprinzipien einer modernen, auf Funktionserhalt aus- gerichteten organerhaltenden Therapie berücksichtigten [1, 8]. Diese Therapiestrategie beinhaltet eine möglichst komplette, aber organerhaltende Tumorresektion (Resektion mit adjuvanter Therapie ist erfolgreicher als alleinige Bestrahlung), danach eine simultane Radiochemotherapie als wichtigster Baustein zur langfristigen Tumorkontrolle (vor allem Cisplatin verstärkt die Strahlenwirkung relevant), anschließend Re-Evaluierung des Therapieansprechens und Beschränkung der Radikal-OP auf Patienten mit Resttumor oder Rezidiv. Die Strahlendosis wird in diesen Konzepten (anders als bei der ,radikalen Radiotherapie“) auf eine moderate, hinsichtlich Funktionserhalt optimale Dosis reduziert. Beim Blasenkarzinom beträgt diese Dosis, die auf die ganze Blase ohne relevantes Risiko für Funktionseinschränkungen appliziert werden kann, etwa 55 bis $60 \mathrm{~Gy}$ in konventioneller Fraktionierung. Das Ziel einer maximalen Tumorkontrolle durch Strahlentherapie wird dabei dem Funktionserhalt untergeordnet. Die auf diese Weise erreichten Überlebensraten mit Funktionserhalt sind gut und beispielsweise besser als beim Larynxkarzinom, bei dem heute ein Stimm- und Kehlkopferhalt angestrebt wird und bei etwa drei Viertel der Patienten auch gelingt. Die simultane Chemotherapie leistet einen wichtigen Beitrag zur lokalen Tumorkontrolle; dies ist auch durch randomisierte Studien bestätigt [5].

Die Zystektomie bedeutet für viele Patienten eine erhebliche Belastung und Einbuße an Lebensqualität und ist aus meiner Sicht in diesem Punkt durchaus einer Laryngektomie vergleichbar, auch wenn die Auswirkungen für $\mathrm{Au}$ Benstehende weniger offensichtlich sind. Die von operativer Seite als Durchbruch gefeierten orthotopen Ersatzblasen kommen nur bei weniger als der Hälfte der Patienten zum Einsatz, und $10 \%$ dieser Patienten sind dann auch noch inkontinent. In der Praxis werden infolge dessen weiterhin landauf landab überwiegend Ableitungen nach außen gelegt, die als „,nasses Stoma“ schwieriger zu pflegen sind als ein Anus praeter. Auch gibt es sekundäre sexuelle Probleme. Ferner ist die Zystektomie per se nicht gerade komplikationslos, und bei fortgeschrittenen Tumoren besteht ein hohes lokoregionales Rezidivrisiko. Gerade für dieses Kollektiv an der Grenze zwischen kurativer Intention und palliativer Therapie sollte deshalb ein organerhaltendes Konzept mit Radiochemotherapie als Option der ersten Wahl gelten.

Das besondere an den hier vorgestellten Erlanger Daten ist der zusätzliche Einsatz der Hyperthermie simultan zur voll dosierten RCT [6]. Die Erlanger Kollegen sind auf diesem Gebiet international führend. Die hier berichteten Daten zum Blasenerhalt sind exorbitant gut und müssen als neuer Benchmark angesehen werden. Ich kenne keine besseren Daten. 


\section{Fazit}

„Are we ignoring the evidence?", fragte schon vor fast 20 Jahren die amerikanische Onkologin Mary Gospodarowicz. Die Antwort lautet leider auch heute noch: „Yes, we do." Das sollte uns allen ein Ansporn sein, für die uns anvertrauten Patienten das Beste zu erreichen.

\section{Jürgen Dunst, Kiel}

Funding Open Access funding provided by Projekt DEAL.

Interessenkonflikt J. Dunst gibt an, dass kein Interessenkonflikt besteht.

Open Access Dieser Artikel wird unter der Creative Commons Namensnennung 4.0 International Lizenz veröffentlicht, welche die Nutzung, Vervielfältigung, Bearbeitung, Verbreitung und Wiedergabe in jeglichem Medium und Format erlaubt, sofern Sie den/die ursprünglichen Autor(en) und die Quelle ordnungsgemäß nennen, einen Link zur Creative Commons Lizenz beifügen und angeben, ob Änderungen vorgenommen wurden.

Die in diesem Artikel enthaltenen Bilder und sonstiges Drittmaterial unterliegen ebenfalls der genannten Creative Commons Lizenz, sofern sich aus der Abbildungslegende nichts anderes ergibt. Sofern das betreffende Material nicht unter der genannten Creative Commons Lizenz steht und die betreffende Handlung nicht nach gesetzlichen Vorschriften erlaubt ist, ist für die oben aufgeführten Weiterverwendungen des Materials die Einwilligung des jeweiligen Rechteinhabers einzuholen.

Weitere Details zur Lizenz entnehmen Sie bitte der Lizenzinformation auf http://creativecommons.org/licenses/by/4.0/deed.de.

\section{Literatur}

1. Efstathiou JA, Spiegel DY, Shipley WU et al (2012) Long-term outcomes of selective bladder preservation by combined-modality therapy for invasive bladder cancer: the MGH experience. Eur Urol 61:705-711

2. Fahmy O, Khairul-Asri MG, Schubert T et al (2018) A systematic review and meta-analysis on the oncological long-term outcomes after trimodality therapy and radical cystectomy with or without neoadjuvant chemotherapy for muscle-invasive bladder cancer. Urol Oncol 36:43-53

3. Gospodarowicz M (2002) Radiotherapy and organ preservation in bladder cancer: are we ignoring the evidence? J Clin Oncol 20:3048-3050

4. https://www.krebshilfe.de/infomaterial/Blaue_Ratgeber/ Blasenkrebs_BlaueRatgeber_DeutscheKrebshilfe.pdf. Zugegriffen: 27.02.2020

5. James ND, Hussain SA, Hall E et al (2012) Radiotherapy with or without chemotherapy in muscle-invasive bladder cancer. N Engl J Med 366:1477-1488

6. Longo TA, Gopalakrishna A, Tsivian M et al (2016) A systematic review of regional hyperthermia therapy in bladder cancer. Int J Hyperthermia 32:381-389

7. Merten R, Ott O, Haderlein M et al (2019) Long-term experience of chemoradiotherapy combinedwith deep regional hyperthermia for organ preservationin high-risk bladder cancer (Ta, Tis, T1, T2). The Oncol 24:1-10

8. Rödel C, Grabenbauer GG, Kühn R et al (2002) Combined-modality treatment and selective organ preservation in invasive bladder cancer: long-term results. J Clin Oncol 20:3061-3071

9. Scrimger RA, Murtha AD, Parliament MB et al (2001) Muscleinvasive transitional cell carcinoma of the urinary bladder: population-based study of patterns of care and prognostic factors. Int J Radiat Oncol Biol Phys 51:23-30 